\title{
Prevalence of Ochratoxin A in Human Milk in the Khorrambid Town, Fars Province, South of Iran
}

\author{
Parvin Dehghan ${ }^{1}$; Keyvan Pakshir ${ }^{2}$; Hossein Rafiei ${ }^{1,}$; Mostafa Chadeganipour ${ }^{1}$; Mojtaba \\ Akbari $^{3}$ \\ ${ }^{1}$ Department of Parasitology and Mycology, School of Medicine, Isfahan University of Medical Sciences, Isfahan, IR Iran \\ ${ }_{2}^{2}$ Department of Medical Mycology and Parasitology, Basic sciences in Infectious Diseases Research Center, School of Medicine, Shiraz University of Medical Sciences, Shiraz, IR Iran \\ ${ }^{3}$ Deputy of Research, School of Medicine, Isfahan University of Medical Sciences, Isfahan, IR Iran \\ ${ }^{*}$ Corresponding author: Hossein Rafiei, Department of Parasitology and Mycology, School of Medicine, Isfahan University of Medical Sciences, Isfahan, IR Iran. Tel: +98-36680042, \\ Fax:+98-36688597, E-mail: rafiei_gh421@yahoo.com
}

Received: March 16, 2013; Revised: June 16, 2013; Accepted: August 4, 2013

\begin{abstract}
Background: Ochratoxins belong to a group of mycotoxins produced as the secondary metabolites by filamentous fungi, such as Aspergillus and Penicillium. These toxins may be teratogenic, mutagenic, hepatotoxic, nephrotoxic, and may have immunosuppressive effects and pose a serious health problems to exposed humans and animals.

Objectives: The present study aimed to determine the level of ochratoxin A (OTA) in the samples of mothers' milk in the Khorrambid Town, Fars Province, south of Iran.

Materials and Methods: Between June and July 2011, samples of human milk were obtained from 87 mothers. The samples were diluted by absolute methanol at 1:4 ratio and after centrifugation, the supernatant was directly used to determine the level of OTA using competitive enzyme-linked immunosorbent assay (ELISA).

Results: Among 87 human milk samples, 84 (96.6\%) samples had positive results for OTA at a mean level of $24.57 \pm 13.6 \mathrm{ng} / \mathrm{L}$. According to the European Union Standard, 14 (16\%) positive samples revealed more than the maximum limit of $40 \mathrm{ng} / \mathrm{L}$ for ochratoxin (range, 1.6-60 $\mathrm{ng} / \mathrm{L})$.

Conclusions: Presence of OTA in the milk of mothers denotes a probable consumption of a contaminated foods. Therefore, regular monitoring of foods for presence of mycotoxins for lactating mothers seems necessary.
\end{abstract}

Keywords:Mycotoxins; Ochratoxin A; Milk; Human

\section{Background}

Breastfeeding is highly important for the infants' growth and health as well as their protection against many diseases. Based on the great advantages of breastfeeding, the World Health Organization (WHO) suggested that the infants should solely be fed by breast milk during the first six months of life (1). Although breast milk feeding has beneficial nutritional and immunological effects, there is the risk of mycotoxins entrance through consumption of the contaminated foods with molds and their metabolites (2). Mycotoxins are toxic secondary metabolites which are produced by filamentous fungi in suitable environmental conditions; thus, fungal growth in feeds should be avoided $(3,4)$.

Ochratoxins are a group of mycotoxins produced by mold fungi particularly Penicillium verrucosum, P. nordicum, Aspergillus ochraceus, A. sulphureus, and A. niger during the storage of cereals and other plant-derived products under non-optimal conditions (5). These fungi can contaminate several kinds of crops, vegetables, and fruits, which are used for human and animal consumption; therefore, their metabolites may be present in all kinds of raw and processed agricultural materials (3, 6, 7). Due to these toxic properties, regulations to control mycotoxins such as ochratoxins have been established in many countries $(3,7,8)$.

In the recent few years there are increasing reports concerning food commodities such as grains, cereals, dried fruits, and milk contamination with ochratoxin A (OTA) (3, 9-13). OTA was shown to have carcinogenic and nephrotoxic effects. It has negative effects on the immune system function leading to teratogenesis and poses damages to DNA. Moreover, this toxin has also been reported to be related to Balkan (endemic) Nephropathy in humans $(14,15)$, and it has been considered as a contaminant of breast milk, as well (16). International Agency for Research on Cancer (IARC) has Finally, the risks of this toxin and its complications should be reduced in humans, especially in infants.

Copyright @ 2014, Ahvaz Jundishapur University of Medical Sciences; Published by Kowsar Corp. This is an open-access article distributed under the terms of the Creative Commons Attribution License, which permits unrestricted use, distribution, and reproduction in any medium, provided the original work is properly cited. 
Table 1. Anthropometric Characteristics of the Mothers and Their Infants and Their Association With the OTA Concentration in Breast Milk Samples ${ }^{\text {a }}$

\begin{tabular}{lccc}
\hline Variable & Mean $\pm \mathbf{S D}$ & \multicolumn{2}{c}{ Ochratoxin A } \\
\cline { 3 - 4 } & & $\mathbf{r}^{\mathrm{b}}$ & P Value \\
\hline Mother's Age, $\mathbf{y}$ & $27.4 \pm 5.18$ & 0.13 & $>0.05$ \\
\hline Mother's Weight, $\mathbf{~ K g}$ & $59.4 \pm 10.34$ & 0.02 & $>0.05$ \\
\hline Mother's Height, $\mathbf{c m}$ & $158.9 \pm 5.4$ & -0.03 & $>0.05$ \\
\hline Mother's BMI & $23.5 \pm 4.04$ & 0.02 & $>0.05$ \\
\hline Infant's Age, mo & $5.26 \pm 2.78$ & -0.02 & $>0.05$ \\
\hline Infants' Weight at Birth, Kg & $3.17 \pm 0.52$ & -0.02 & $>0.05$ \\
\hline $\begin{array}{l}\text { Infants' Height at Birth, cm } \\
\text { Infants' Head }\end{array}$ & $49.21 \pm 3.46$ & -0.04 & 0.07 \\
\hline $\begin{array}{l}\text { Circumference at Birth, cm } \\
\text { a Abbreviation: BMI, body mass index. }\end{array}$ & $34.73 \pm 1.66$ & -0.25 & $>0.05$ \\
b Spearman Correlation Coefficient. & & \\
\hline
\end{tabular}

classified OTA in 2B category as a probable reason for human cancer (17).

In comparison to the adults, infants are more sensitive to the effects of mycotoxins because they have a lower weight but a higher metabolic rate. In addition, due to the incomplete growth of some of their organs and tissues, particularly the central nervous system, they have lower capability for neutralizing the toxins entering the body $(6,18)$. Best on our knowledge and after a comprehensive review of the literature, there was no study to determine the level of OTA in milk samples of Iranian mothers. Therefore, the present study was undertaken to determine the level of OTA in breast milk samples in Southern Iran.

\section{Objectives}

The natural prevalence of OTA in human milk from Iran had not been studied; hence, we aimed to determine the level of OTA in the mothers' milk samples in the town of Khorrambid, Fars Province, south of Iran.

\section{Materials and Methods}

In the current study, 87 breast milksamples were collected from seven health centers of Khorrambid Town from June to July 2011. They were evaluated for presence of OTA. The inclusion criteria were being a healthy breast feeder and having an infant younger than one year old. After making the necessary arrangements, the mothers were summoned to the health centers in the morning and were informed about the research objectives. A written informed consents was signed by participants and 5 to $10 \mathrm{~mL}$ of breast milk sample was collected in the sterile bottles. The samples were kept at $-20^{\circ} \mathrm{C}$ until measurement processes. Before starting the experiments, the samples were thawed. OTA level was determined in breast milks using a competitive enzyme-linked immunosorbent assay (ELISA) method (Helica Company, Santa Ana, California, USA).
According to the kit procedure, the breast milk samples were diluted by absolute methanol at 1:4 ratio and kept at room temperature for five minutes. They were centrifuged at $\times 3500 \mathrm{~g}$ and $10^{\circ} \mathrm{C}$ for 15 minutes. The supernatant was used to determine the concentration of OTA. The required number of Microtiter wells was located in the plate, the standard solutions and the breast milk samples were added to the wells, and washed after each incubation session. All steps were performed according to the manufacturer's instruction. The optical absorption of the samples was measured through the photometric method using ELISA reader at the wavelength of $450 \mathrm{~nm}$. Afterwards, the calibration curve was drawn and used in order to determine the OTA concentration considering the samples' absorption rate. Data were analyzed using SPSS version Version 16 (SPSS Inc., Chicago, IL, USA) employing Mann-Whitney U test, Kruskal-Wallis test, and correlation coefficient.

\section{Results}

The demographic data of the 87 mothers and their infants are presented in Table 1. Calculation of body mass index (BMI) showed that $11.5 \%, 28.7 \%$, and $5.7 \%$ of the mothers under study were underweight, overweight, and obese, respectively, while $54 \%$ had normal BMI. Moreover, 55 (63.2\%) and 52 (59.8\%) mothers were involved in farming and ranching, respectively. Twenty subjects (23\%) had miscarriage. Overall, 57 (65.5\%), 24 (27.6\%), and 6 (6.9\%) mothers had under diploma, diploma, and above diploma education levels, respectively. Among the 87 breast milk samples, 84 mothers had mean OTA of $24.57 \pm 13.6$ $\mathrm{ng} / \mathrm{L}$ (range, $1.6-60 \mathrm{ng} / \mathrm{L}$ ).

The results of Mann-Whitney U nonparametric test showed no significant association between OTA concentration and ranching $(P>0.05)$. Regarding OTA concentration, no significant difference was observed between the mothers who were involved in farming and those who were not $(\mathrm{P}>0.05)$. Moreover, no significant association was noticed between the OTA concentration and miscarriage $(P>0.05)$. The results of Kruskal-Wallis nonparametric test revealed a statistically significant association between the mothers' level of education and OTA concentration $(\mathrm{P}=0.05)$. Furthermore, The OTA concentration of mothers' milk in the villages where the sampling was done, was different from each other and it showed that OTA concentration was significantly associated with the residing villages of mothers $(\mathrm{P}=0.039)$.

\section{Discussion}

In suitable conditions, various fungi such as Aspergillus, Penicillium, and Fusarium can easily contaminate the agricultural products during planting, growing, and harvesting (5). By consuming the foodstuff contaminated with the fungi producing such toxins, humans and animals are exposed to contamination with these mycotoxins $(5,19)$. Humans are contaminated 
with mycotoxins, such as OTA through different routes. Measurement of these toxins in the biological fluids of the body is a valuable index for examining the actual contamination with such toxins (20).

Since breast milk is a unique source for the infants' nutrition and healthy growth, breastfeeding has been emphasized and encouraged all over the world. Therefore, mothers may face different natural or artificial contaminants and foodstuffs contaminated with various amounts of toxins during breastfeeding. Hence, consumption of healthy natural foodstuffs is of great importance for humans, particularly breastfeeding mothers (21).

Various reports from different countries revealed that human milk may contain OTA with different concentrations. In the present study, 84 (96.6\%) out of the 87 breast milk samples were contaminated with OTA. The result of a similar study in Egypt indicated that from a total 120 human milk samples, OTA was found in 43 (35.8\%) of the samples (22). In addition, the mean concentration of the toxin in this study was higher than that of the previous studies, such as $17.5 \mathrm{ng} / \mathrm{L}$ in Brazil (12), 10-57 ng/L in Italy (23,24), and 17-30 ng/L in Germany (16), while lower amounts such $8.87 \mathrm{ng} / \mathrm{L}$ was noticed in Egypt (25), 106 $\mathrm{ng} / \mathrm{L}$ in Chile (26), $39.8 \mathrm{ng} / \mathrm{L}$ in Norway (27), and $7900 \mathrm{ng} / \mathrm{L}$ in Sierra Leone (28).

Moreover, OTA concentration in our study ranged from 1.6 to $60 \mathrm{ng} / \mathrm{L}$, which is in agreement with the study performed in Slovakia (range, 2.3-60.3 ng/L). Nevertheless, the contamination rate was $96.6 \%$ in the current study, while it was reported as $30.2 \%$ in the Slovakian study (9). Up to now, a large number of investigations have been performed on the risks of consuming OTA and different values of tolerable daily intake (TDI) have been suggested $(11,18,29)$. For instance, Nordic Working Group has suggested the highest daily TDI of OTA level in human's body as $5 \mathrm{ng} / \mathrm{kg}$ of baby weight (30). Recently, based on the regulations made by the European Union's cooperating with European Food Safety Authority (EFSA), the tolerable weekly intake of OTA has been reported as 120 $\mathrm{ng} / \mathrm{kg}$ body weight and TDI of $20 \mathrm{ng}$ has been suggested for the children (23).

In the current study, 14 (16\%) out of the 84 (96.6\%) positive samples showed more than the maximum limit of $40 \mathrm{ng} / \mathrm{L}$ of OTA. Assuming that an infant is $4 \mathrm{~kg}$ and consumes $500 \mathrm{~mL}$ milk every day, no more than $40 \mathrm{ng} / \mathrm{L}$ OTA must be present in the milk; however, the results of this study showed that $16 \%$ of the infants were exposed to the milk contaminated by more than permitted TDI of OTA. Although the short-term side effects of OTA have not been well identified in humans, the continuous absorption of this mycotoxin in the body and its long half-life in the blood (35 days) lead to accumulation of a large amount of this toxin in the infant's body, which results in adverse effects over time (31). Recent studies on detecting of OTA in beans and dried fruits in Iran indicate that from 30 bean samples, three samples contained this toxin with the mean value of $0.29 \mathrm{ng} / \mathrm{g}$; in addition, OTA was detected in $3.33 \%$ and $20 \%$ of dried apricots and prunes, respectively $(10,13)$.

It seems that the environmental factors, such as temperature and humidity as well as the way that the foodstuffs and agricultural products are kept in this region, are the main reasons for high concentration of OTA in the mothers' milk. In fact, most of the people in the studied area have traditional lifestyles and they produce and preserve most of their necessary foodstuffs and agricultural products for a long time. The study results confirmed the existence of OTA in the breast milk of the mothers and consumption of the foodstuffs contaminated with such toxins. These toxins have long-term side effects; however, mothers are not recommended to stop breastfeeding their infants, as the advantages of breastfeeding are clear.

In conclusion, comprehensive programs should be developed in order to regularly investigate and control these toxins in both humans' and animals' food chains so that the amount of these toxins can be reduced and their side effects can be prevented. Moreover, further studies in other parts of the country are recommended in order to identify the status of the society members' exposure to these toxins. Monitoring of foods for the presence of mycotoxins like OTA and disposal of contaminated products should decrease the risk to the human and animal health. Therefore, regular monitoring of foods for presence of mycotoxins for lactating mothers seems necessary.

\section{Acknowledgements}

The present article was adopted from proposal No. 390272 approved by the Department of Parasitology and Mycology, Isfahan University of Medical Sciences, Isfahan, Iran. Hereby, the authors would like to thank Isfahan University of Medical Sciences, Shiraz University of Medical Sciences, and the personnel of the health and treatment network of Khorrambid Town for their collaboration in performing the research.

\section{Authors' Contributions}

Study concept and design: Rafiei, Dehghan, and Pakshir. Acquisition of data and sampling: Rafiei. Analysis and interpretation of data: Rafiei, Dehghan, and Akbari. Drafting of the manuscript: Rafiei, Dehghan, Pakshir and Chadeganipour. Critical revision of the manuscript for important intellectual content: Rafiei, Dehghan, Pakshir, and Chadeganipour. Statistical analysis: Rafiei and Akbari. Study supervision: Dehghan and Pakshir.

\section{Financial Disclosure}

Isfahan University of Medical Sciences.

\section{Funding/Support}

Isfahan University of Medical Sciences. 


\section{References}

1. WHO.. Global strategy for infant and young child feeding.Geneva: World Health Organization; 2003.

2. Jensen AA. Levels and trends of environmental chemicals in human milk. In: Jensen AA, Slorach SA editors. Chemical contaminants in human milk.;1991. pp. 45-198.

3. Jorgensen K, Rasmussen G, Thorup I. Ochratoxin A in Danish cereals 1986-1992 and daily intake by the Danish population. Food Addit Contam. 1996;13(1):95-104.

4. Speijers GJA, Van Egmond HP. Worldwide ochratoxin A levels in food and feeds.: Colloques-Institut National De La Sante Et De La Recherche Medicale Colloques Et Seminaires; 1993.

5. Samson RA, Hoekstra ES, Frisvad JC. Introduction to food-and airborne fungi.: Centraalbureau voor Schimmelcultures; 2004.

6. Galvano F, Galofaro V, Galvano G. Occurrence and stability of aflatoxin M1 in milk and milk products: a worldwide review. J Food Protect.1996;59(10):1079-90.

7. Zain ME. Impact of mycotoxins on humans and animals. J Saudi Chem Soc. 2011;15(2):129-44.

8. van Egmond HP, Schothorst RC, Jonker MA. Regulations relating to mycotoxins in food: perspectives in a global and European context. Anal Bioanal Chem. 2007;389(1):147-57.

9. Dostal A, Jakusova L, Cajdova J, Hudeckova H. Results of the first studies of occurence of ochratoxin A in human milk in Slovakia. Bratisl Lek Listy. 2008;109(6):276-8.

10. Fakoor Janati SS, Beheshti HR, Khoshbakht Fahim N, Feizy J. Aflatoxins and ochratoxinin A in bean from Iran. Bull Environ Contam Toxicol. 2011;87(2):194-7.

11. Joint FAO/WHO Expert Committee on Food Additives. Meeting World Health Organization .. Evaluation of Certain Food Additives and Contaminants: Sixty-eighth Report of the Joint FAO/WHO Expert Committee on Food Additives.: World Health Organization; 2007.

12. Navas SA, Sabino M, Rodriguez-Amaya DB. Aflatoxin M(1) and ochratoxin A in a human milk bank in the city of Sao Paulo, Brazil. Food Addit Contam. 2005;22(5):457-62.

13. Janati SS, Beheshti HR, Asadi M, Mihanparast S, Feizy J. Preliminary survey of aflatoxins and ochratoxin a in dried fruits from Iran. Bull Environ Contam Toxicol. 2012;88(3):391-5.

14. Galvano F, Ritieni A, Piva G, Pietri A, Diaz D. Mycotoxins in the human food chain.: Mycotoxin Blue Book; 2005.

15. Sauvant C, Holzinger H, Mildenberger S, Gekle M. Exposure to nephrotoxic ochratoxin A enhances collagen secretion in human renal proximal tubular cells. Mol Nutr Food Res. 2005;49(1):31-7.

16. Gareis M, Martlbauer E, Bauer J, Gedek B. [Determination of ochratoxin A in human milk].Z Lebensm Unters Forsch. 1988;186(2):114-7.
17. Pfohl-Leszkowicz A, Manderville RA. Ochratoxin A: An overview on toxicity and carcinogenicity in animals and humans. Mol Nutr Food Res. 2007;51(1):61-99.

18. Kuiper-Goodman T. Risk assessment of ochratoxin A: an update. Food Addit Contam. 1996;13 Suppl:53-7.

19. Sherif SO, Salama EE, Abdel-Wahhab MA. Mycotoxins and child health: the need for health risk assessment. Int J Hyg Environ Health. 2009;212(4):347-68.

20. el-Nezami HS, Nicoletti G, Neal GE, Donohue DC, Ahokas JT. Aflatoxin M1 in human breast milk samples from Victoria, Australia and Thailand. Food Chem Toxicol.1995;33(3):173-9.

21. Gurbay A, Sabuncuoglu SA, Girgin G, Sahin G, Yigit S, Yurdakok $\mathrm{M}$, et al. Exposure of newborns to aflatoxin M1 and B1 from mothers' breast milk in Ankara, Turkey. Food Chem Toxicol. 2010;48(1):314-9.

22. El-Sayed AA, Soher EA, Neamat-Allah A. Human exposure to mycotoxins in Egypt. Mycotoxin Res. 2002;18(1):23-30.

23. Biasucci G, Calabrese G, Di Giuseppe R, Carrara G, Colombo F Mandelli B, et al. The presence of ochratoxin A in cord serum and in human milk and its correspondence with maternal dietary habits. EurJ Nutr. 2011;50(3):211-8.

24. Turconi G, Guarcello M, Livieri C, Comizzoli S, Maccarini L, Castellazzi AM, et al. Evaluation of xenobiotics in human milk and ingestion by the newborn--an epidemiological survey in Lombardy (Northern Italy). Eur J Nutr. 2004;43(4):191-7.

25. El-Sayed Abd Alla A, Neamat-Allah A, Aly SE. Situation of mycotoxins in milk, dairy products and human milk in Egypt. Mycotoxin Res. 2000;16(2):91-100.

26. Munoz K, Campos V, Blaszkewicz M, Vega M, Alvarez A, Neira J, et al. Exposure of neonates to ochratoxin A: first biomonitoring results in human milk (colostrum) from Chile. Mycotoxin Res. 2010;26(2):59-67.

27. Creppy EE. Update of survey, regulation and toxic effects of mycotoxins in Europe. Toxicol Lett. 2002;127(1-3):19-28.

28. Jonsyn FE, Maxwell SM, Hendrickse RG. Ochratoxin A and aflatoxins in breast milk samples from Sierra Leone. Mycopathologia. 1995;131(2):121-6.

29. Kuiper-Goodman T, Scott PM. Risk assessment of the mycotoxin ochratoxin A. Biomed Environ Sci.1989;2(3):179-248.

30. Thuvander A, Paulsen JE, Axberg K, Johansson N, Vidnes A, Enghardt-Barbieri $\mathrm{H}$, et al. Levels of ochratoxin A in blood from Norwegian and Swedish blood donors and their possible correlation with food consumption. Food Chem Toxicol.2001;39(12):1145-51.

31. Skaug MA, Stormer FC, Saugstad OD. Ochratoxin A: a naturally occurring mycotoxin found in human milk samples from Norway. Acta Paediatr. 1998;87(12):1275-8. 